\title{
Analysis of Thermal Conductivity of Frost on Cryogenic Finned-tube Vaporizer Using Fractal Method
}

\author{
Shuping Chen, Shuting Yao, Fushou Xie \\ School of Petrochemical Engineering, Lanzhou University of Technology, Lanzhou, China \\ Email: chensp@lut.cn
}

Received March, 2013

\begin{abstract}
Thermal conductivity of frost is not only related to density, but also affected by its microstructure and environmental conditions, and it will continuously change with the formation and growth of frost. Images of frost formation and growth on the cryogenic surface in various shapes at different stages were obtained by experimental measurements, and a numerical simulation of frost formation and growth was carried out based on Diffusion Limited Aggregation (DLA) model of fractal theory in this paper. Based on the frost structure obtained by experiment, the fractal dimension of pore area distribution and porosity of frost layer on the cryogenic finned-tube vaporizer were calculated by using fractal method, and combined with heat conduction model of frost layer obtained by thermal resistance method, the thermal conductivity of frost on the cryogenic surface was calculated. The result shows that the thermal conductivity calculated by the fractal model coincides with the range of the experimental data. Additionally, comparison with other heat conduction models indicated that it is feasible to introduce the fractal dimension of pore area distribution into heat conduction model to deduce the thermal conductivity of frost.
\end{abstract}

Keywords: Cryogenic Finned-tube Vaporizer; Thermal Conductivity; Frost Layer; Fractal

\section{Introduction}

Finned-tube vaporizer will be frosted once its surface temperature is below the dew point of surrounding humid air, and lower than $0^{\circ} \mathrm{C}$. The frost on the surface of vaporizer will decrease the efficiency of vaporizer, increase the pressure loss and even result in the failure of the vaporizer system. Sublimation frosting of humid air on the vaporizer surface involves heat transfer, mass transfer and the boundary movement of special porous medium. Of all the issues concerning the sublimation frosting, frost physical properties especially the thermal conductivity is one of the key parameters. There have been a lot of researches on the effect of thermal conductivity on frosting problem. At the beginning, researchers mainly concentrated on the experimental study of the formation and growth of frost on the simple geometric surfaces, such as level plate, pipe and vertical plate. Various empirical or semi-empirical correlations of the density and thermal conductivity were proposed [1-7]. Then, numerous attempts on the growth and physical properties of frost on the cold plate or pipe based on the mechanism of frosting were made, too. Based on the mechanism of frosting, and considering the process of water vapor diffusion in frost, Brain [8] firstly put forward that water vapor flowing into the frost was divided into two parts, one part was used to increase the thickness of frost, the other part to increase the density of frost. Hayashi Y [9] believed that the change of density essentially depended on the surface temperature of frost which was changing frequently in the process of frosting. According to the different structures at different growth and formation stages of frost, he demonstrated three periods which could describe the evolution of frost layer: the crystal growth period, the frost layer growth period, and the frost full-growth period, and finally proposed the well-known correlation for frost density in terms of frost temperature and ice-air mixture model. On the basis of the theory of Hayashi Y, considering the space distribution and time-varying of density and temperature of frost, Tao Y $X$ [10] applied the control volume average method of porous medium into ice-air mixture model and improved the accuracy of the model. Le Call R [11] modified the boundary conditions and the permeability coefficient of water vapor in Tao Y X model, and focused on the effective diffusion coefficient of water vapor, then, he got the distribution of density, temperature, thermal conductivity in frost. Chen H [12] further modified Tao Y X model, and set up a mathematic model of dynamic growth characteristics of frost on a flat plate.

In these researches, the frost was considered as a virtual continuous medium which was distributed uniformly on a large scale, that is, the volume averaging theory was 
used to describe the geometric distribution of frost. However, as a porous medium, the thermal conductivity of frost is not only related to density, but also affected by its microstructure and the environmental conditions. The thermal conductivity will continuously change with the formation and growth of frost, therefore, the thermal conductivity is a function of density, porosity, tortuosity and other parameters. The over-simplified frosting model will result in a serious error. The formation and growth of frost is a complicated process, therefore, it is very difficult to obtain an equation of thermal conductivity based on a theory. Based on simulating the frost crystal structure which has fractal growth characteristics, Cai L [13] set up an equilibrium equation at each node and predicted the thermal conductivity of frost by using the DLA model. In the present paper, on the basis of the previous work, according to the experimental observation of frost formation and growth, the physical process of frost growth was simulated by DLA (Diffusion Limited Aggregation) model of fractal theory. The box counting dimension method was used to determine the fractal dimension of pore area distribution and fractal porosity of frost profile. Thereby, a fractal model of heat conduction in frost layer was established theoretically to determine the effective thermal conductivity.

\section{Frost Formation and Growth Process}

The experimental facility utilized in this work is shown in Figure 1. The facility is composed of the feeding liquid system, the vertical frosting surface, the image acquisition system and the data acquisition system. This experiment is conducted under the conditions of ambient temperature $T_{\infty}=16.3{ }^{\circ} \mathrm{C}$, humidity $R H=55 \%$, and these environmental parameters remain unchanged during the experiment. As shown in Figure 1, liquid nitrogen flows from the liquid nitrogen tank, then through the vacuum thermal insulating tube to cryogenic vessel. The vacuum thermal insulating tube minimizes the loss of refrigeration capacity before liquid nitrogen flowing into the cryogenic vessel. Cryogenic vessel is welded by aluminum flat plates, wrapped with the polyurethane foam material around it as the thermal insulation layer. A boss (with length of $120 \mathrm{~mm}$, width of $100 \mathrm{~mm}$, the height of $100 \mathrm{~mm}$ ) is extended into the cryogenic vessel, and a vertical flat plate (with length of $120 \mathrm{~mm}$, width of 100 $\mathrm{mm}$ ) in the boss for frosting. Tubing is installed in the bottom of cryogenic vessel for connecting the cryogenic vessel and a glass level gauge, which is used to measure the height of the liquid nitrogen in the cryogenic vessel. Liquid nitrogen in cryogenic vessel absorbs heat by convection between the vertical flat plate and indoor humid air, and then gasifies; humid air will form frost on the surface of vertical flat plate.
Figure 2 shows the experimental images of frost growth on the cryogenic surface (with cryogenic surface temperature $T_{\mathrm{w}}=-120^{\circ} \mathrm{C}$ ) at different time, it can be seen from (a) and (b) that many dendritic frost crystals formed on the cryogenic surface, and new dendritic crystals produced with increasing time; as shown in (c), these dendritic frost crystals are characterized with fractal self-similarity to some extent $[14,15]$.

Figure 3 shows the simulated image of frosting on the cryogenic surface of finned-tube vaporizer in MATLAB based on the DLA model of fractal theory. The detailed algorithm of simulating frosting and simulation results have been reported previously [16].

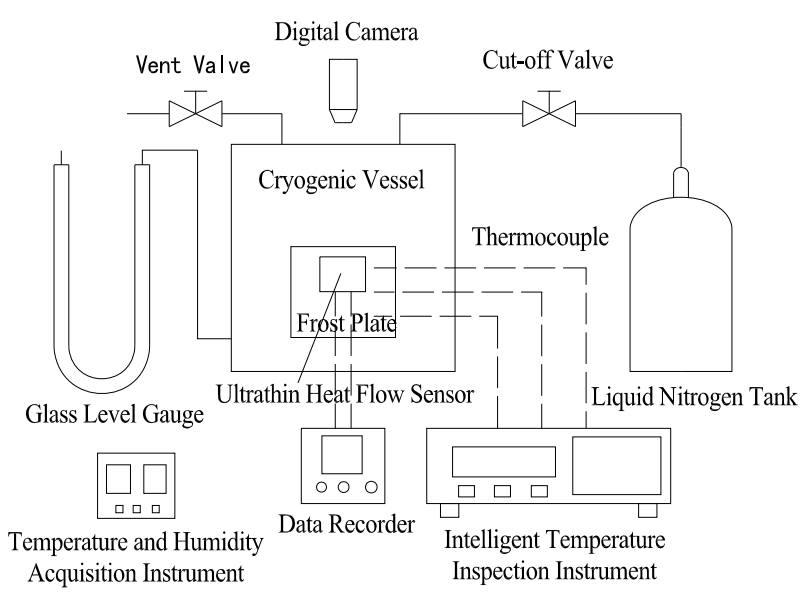

Figure 1. Experimental apparatus for frosting test..

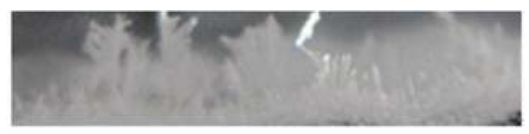

(a) $30 \mathrm{~min}$

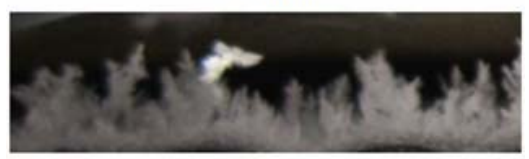

(b) $45 \mathrm{~min}$

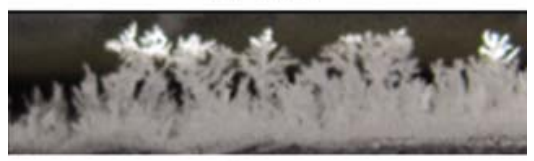

(c) $52 \mathrm{~min}$

Figure 2. Experimental images of frosting on the cryogenic surface.

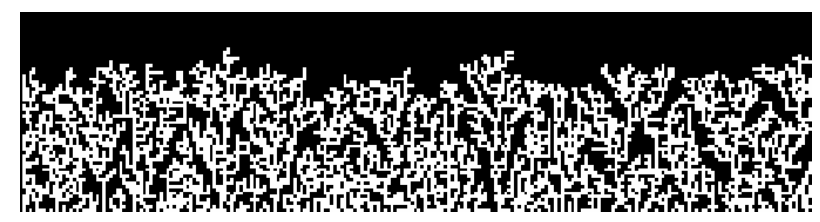

Figure 3. Frosting simulated image on the cryogenic surface of finned-tube vaporizer. 


\section{Fractal Characteristics of Frost Layer}

\subsection{Fractal Dimension}

Taking irregular geometric shape as the research object, fractal geometry is put forward to describe irregular shape with self-similarity. The so-called fractal is the geometric object which has self-similarity in shape and structure, the mathematical expression of fractal is given by

$$
N(\delta) \propto \delta^{\mathrm{d}}
$$

Here, $N$ is occupation space of a fractal object (the line, the area or volume), $\delta$ is measurement scale, and $d$ is fractal dimension, it may be an integer or not an integer. Fractal dimension is the measurement of the complexity of fractal object, a quantitative parameter describing fractal characteristics of an object. The larger value is, the more complex the fractal object is. For a fractal area with $d$ between 1 and 2, its value can be determined by the slope of curve in figure $\ln N(\delta)-\ln \delta$ [17]. Fractal structure is actually an ideal model and is an abstract of many complex objects in nature. The true object in nature is closer to random fractal, that is, random fractal has self-similarity only in a certain scale range, called statistical self-similarity. For example, the self-similarity of porous medium is valid just in a certain scale.

Statistical self-similarity is usually called local fractal. It has been proved that effective thermal physical parameter $E$ of porous medium is not only related to the medium's property of its composition, but also depended on the space structure of porous medium. According to local fractal theory, if a porous medium has fractal structure and local fractal scale $l$, the effective thermal physical parameter of porous medium can be expressed as

$$
E=f\left(\sum E_{i}, \varepsilon, d, l\right)
$$

where $E_{\mathrm{i}}$ is thermal physical parameter of each phase composed of porous medium, $\varepsilon$ is average volume porosity of porous medium, $d$ is fractal dimension. This formula is the basic equation of determining thermal physical parameter of fractal object based on the fractal theory.

Frosting is a transient process accompanied by airsolid phase change, related with heat transfer, mass transfer, and the boundary movement of porous medium. Frost layer may be considered as a random porous medium consisting of ice and air, just as shown in Figure 2. Ice crystals skeleton of frost layer or pore is not uniformly distributed on the profile surface, as it is hard to fill the entire profile of frost layer. In many cases, the area distribution of pore structure or ice crystals skeleton has fractal characteristics, therefore, the corresponding fractal dimension of area distribution for pore or ice crystals skeleton can be calculated. The specific calcula- tion procedures are as follows:

1) As shown in Figure 4, taking any different measurement values $X$ in $D \sim A$ interval.

2) Taking a point as the center of calculated area in frost layer profile, the box with sides $X$ is used for measuring the area of pore in the calculated profile.

3) Repeat step (2) many times, until the whole frost layer profile is measured with equal probability, and the average value of pore area is $S$.

4) Change scale value $X$, and repeat the step (2) and step (3), then a series of pore areas $S_{\mathrm{i}}$ corresponding with different scales $X_{\mathrm{i}}$ can be obtained.

5) Using the least square method for linear fitting, the $S$ - $X$ logarithm coordinates figure can be obtained.

It is clear that the average value $S$ of pore area of frost on the cryogenic surface and measurement scale $X$ fit a linearity in the $S-X$ logarithm coordinates figure, and also has the following linear relation:

$$
\operatorname{Ln} S=\ln k+d \ln X
$$

where $k$ is a proportional constant, the slope $d$ is the fractal dimension of pore area distribution of frost layer.

\subsection{Fractal Porosity of Frost Layer}

For frost structure with certain porosity, as the pore area distribution of frost profile has fractal characteristics, the Equation (3) can be rearranged to:

$$
S=k X^{d}
$$

where $d$ is fractal dimension of pore area distribution and $S$ is a measured average value.

By simplifying the actual irregular structure of frost and assuming that the pore structure of frost as a cube, where there is the cavity for air flowing through in the middle of the cubical structure of frost, the rest of the cubical structure is considered as frost ice crystals skeleton. Each calculation volume unit of frost layer is shown in Figure 5. Since the similar structure has an invariant property of scale, the heat transfer characteristics of every calculation volume unit of frost layer will keep in agreement with the whole frost crystal. Then the effective volume porosity of frost based on the fractal theory is defined as:

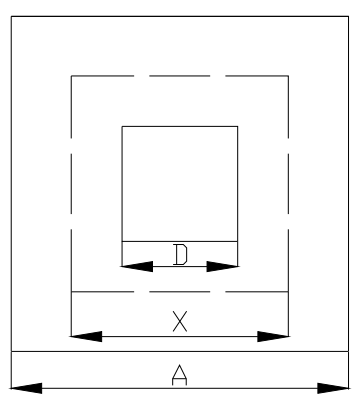

Figure 4. Schematic diagram of measurement scales. 


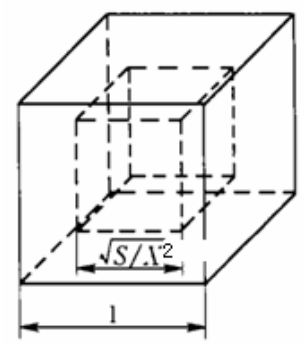

Figure 5. Model of volume unit of frost layer.

$$
\varepsilon=\left(\frac{S}{X^{2}}\right)^{\frac{3}{2}}
$$

Substituting Equation (4) and Equation (5), then:

$$
\rho=\varepsilon \rho_{a}+(1-\varepsilon) \rho_{i c e}
$$

The density of frost based on the fractal porosity can be obtained by the reference [18]:

$$
\varepsilon=k^{\frac{3}{2}} X^{\frac{3}{2}(d-2)}
$$

where $\rho$ is frost density, $\mathrm{kg} \cdot \mathrm{m}^{-3} ; \rho_{\mathrm{a}}$ is air density, $\rho_{\mathrm{a}}$ $=1.292 \mathrm{~kg} \cdot \mathrm{m}^{-3} ; \rho_{\text {ice }}$ is ice density, $\rho_{\text {ice }}=0.9 \times 10^{3} \mathrm{~kg} \cdot \mathrm{m}^{-3}$.

\subsection{Thermal Conductivity of Frost}

As a special porous medium, the actual microstructure of frost is changeable and irregular. The local area fractal, however, still exists from the above analyses. Therefore, a simplified unit pore profile model of frost layer shown in Figure 6. The simplified profile has the same pore area and the same fractal dimension $d$ of local pore area with the original actual profile, and then they should have the same effective thermal properties. For simplifying the calculation, the simplified fractal model of heat conduction in frost layer shown in Figure $\mathbf{7}$ is adopted.

The thermal resistance $R$ of the fractal model is shown in Figure 8 and expressed as:

$$
R=\frac{R_{i 1} \cdot\left(R_{i 2}+R_{a}\right)}{R_{i 1}+R_{i 2}+R_{a}}
$$

where

$$
\begin{gathered}
R_{i 1}=\frac{1-\varepsilon^{\frac{1}{3}}}{\lambda_{i} \cdot\left(1-\varepsilon^{\frac{2}{3}}\right)} \\
R_{i 2}=\frac{\varepsilon^{\frac{1}{3}}\left(1-\varepsilon^{\frac{1}{3}}\right)}{\lambda_{i} \cdot\left(1-\varepsilon^{\frac{2}{3}}\right)} \\
R_{a}=\frac{1}{\lambda_{a} \cdot \varepsilon^{\frac{2}{3}}} \\
R_{i 2}+R_{a}=\frac{\lambda_{a} \cdot \varepsilon \cdot\left(1-\varepsilon^{\frac{1}{3}}\right)+\lambda_{i} \cdot\left(1-\varepsilon^{\frac{2}{3}}\right)}{\lambda_{a} \cdot \lambda_{i} \cdot \varepsilon^{\frac{2}{3}} \cdot\left(1-\varepsilon^{\frac{2}{3}}\right)}
\end{gathered}
$$

Then

$$
R=\frac{\lambda_{i} \cdot \varepsilon \cdot\left(1-\varepsilon^{\frac{1}{3}}\right)^{2}+\lambda_{a} \cdot\left(1-\varepsilon^{\frac{1}{3}}\right)\left(1-\varepsilon^{\frac{2}{3}}\right)}{\lambda_{a} \cdot \lambda_{i} \cdot\left(1-\varepsilon^{\frac{2}{3}}\right) \cdot\left(1-\varepsilon^{\frac{1}{3}}\right) \cdot\left(\varepsilon^{\frac{2}{3}}+\varepsilon\right)+\lambda_{a}^{2} \cdot\left(1-\varepsilon^{\frac{2}{3}}\right)^{2}}
$$

The effective thermal conductivity of frost can be expressed as:

$$
\lambda=\frac{1}{R}=\frac{\lambda_{a} \cdot \lambda_{i} \cdot\left(1-\varepsilon^{\frac{2}{3}}\right) \cdot\left(1-\varepsilon^{\frac{1}{3}}\right) \cdot\left(\varepsilon^{\frac{2}{3}}+\varepsilon\right)+\lambda_{a}^{2} \cdot\left(1-\varepsilon^{\frac{2}{3}}\right)^{2}}{\lambda_{i} \cdot \varepsilon \cdot\left(1-\varepsilon^{\frac{1}{3}}\right)^{2}+\lambda_{a} \cdot\left(1-\varepsilon^{\frac{1}{3}}\right)\left(1-\varepsilon^{\frac{2}{3}}\right)}
$$

where $\lambda_{\mathrm{a}}$ is the thermal conductivity of air, $\lambda_{\mathrm{a}}=0.024$ $\mathrm{W} \cdot \mathrm{m}^{-1} \cdot \mathrm{K}^{-1} ; \lambda_{\mathrm{i}}$ is the thermal conductivity of ice, $\lambda_{\mathrm{i}}=1.88$ $\mathrm{W} \cdot \mathrm{m}^{-1} \cdot \mathrm{K}^{-1} ; \lambda$ is the thermal conductivity of frost.

\section{Results and Discussion}

\subsection{Discussion of Fractal Dimension}

The box counting dimension method is used to calculate the fractal dimension $d$ of pore area distribution of frost for frosting simulation image shown in Figure 3, and then being compared with the fractal dimension of experimental binary image shown in Figure 9. The calculation result is shown in Figure 10. The fractal dimension

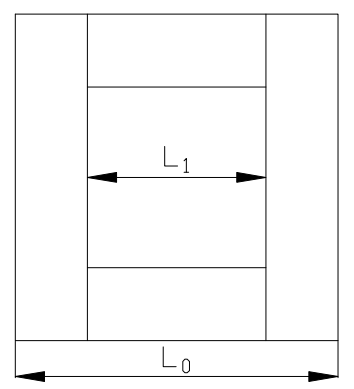

Figure 6. Schematic diagram of an unit pore profile.
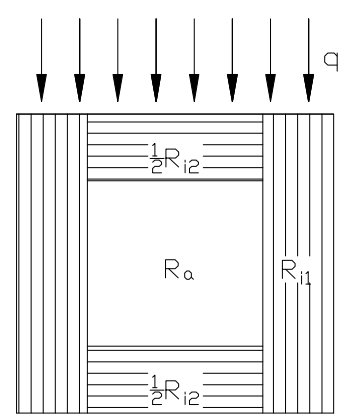

Figure 7. Simplified heat conduction model of frost layer.

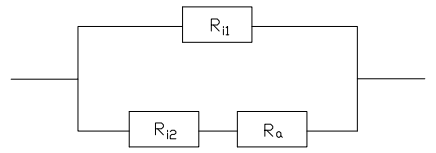

Figure 8. The schematic diagram of total thermal resistance. 


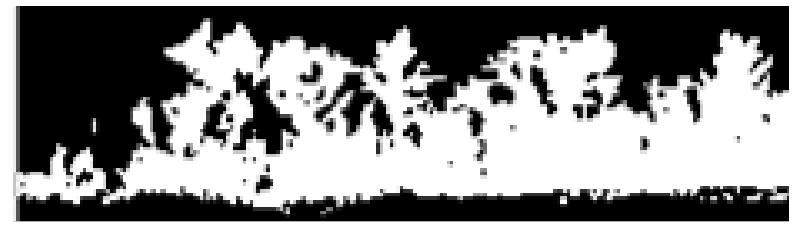

Figure 9. Experimental frosting binary image on the cryogenic surface of finned-tube vaporizer.

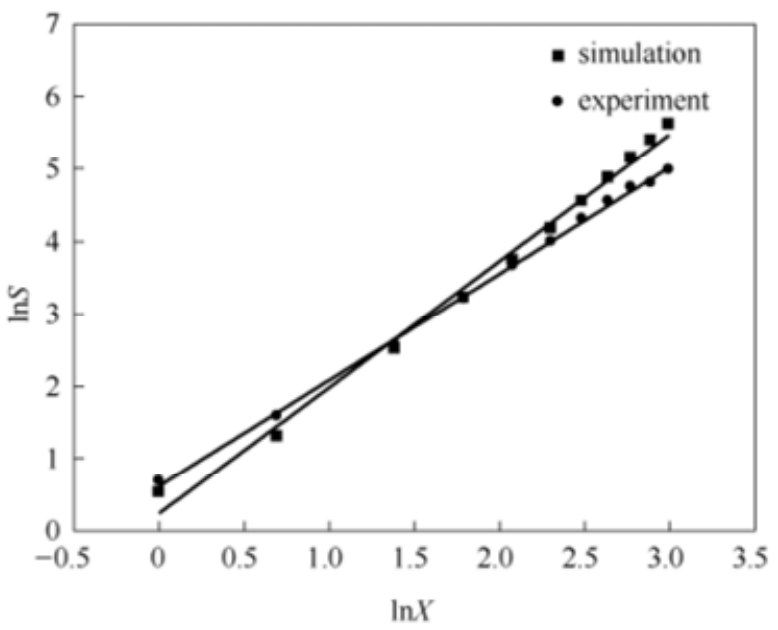

Figure 10. Linear fitting for the fractal dimension of pore area distribution.

of pore area distribution by simulation of frost is $d_{1}=$ 1.743 , and the fractal dimension of pore area distribution from experimental frost is $d_{2}=1.466$. It can be seen from Figure 10 that the profile pore distribution of frost on the surface of finned-tube vaporizer satisfies statistical self-similarity in measurement scale $D \sim A$ interval, namely, the pore distribution of frost profile presents fractal characteristics. In addition, the consistent of the fractal dimension of the simulation image $d_{1}$ with that of the experimental image $d_{2}$ shows the rationality of the numerical simulation and provides a powerful evidence for further deriving the fractal model of heat conduction in frost layer.

\subsection{Thermal Conductivity of Frost Layer}

It can be obtained that the diameter scale of pore space of the actual frost layer is 1 20. In order to establish a general fractal model of frost, a range of $1 \sim 20$ is used as the value of measurement scale $X$ in Figure 10. As shown in Figure 10, $d_{1}=1.743, k_{1}=1.274, d_{2}=1.466, k_{2}=1.861$, substituting the related values into Equation (7), the density $\rho$ of the actual frost can be calculated, as shown in Figure 11. Then the thermal conductivity of actual frost is got by Equation (14), shown in Figure 12. It can be seen that the thermal conductivity of actual frost calculated by the above fractal model agrees well with the range of $0.02 \sim 0.16 \mathrm{~W} \cdot \mathrm{m}^{-1} \cdot \mathrm{K}^{-1}$ of the thermal conduc- tivity reported by reference, and thus explains the rationality of the fractal model of heat conduction.

Figure 13 shows comparison between the proposed model and other models. It has been generally accepted that the thermal conductivity of frost is considered to be depended on density. In fact, there are large differences

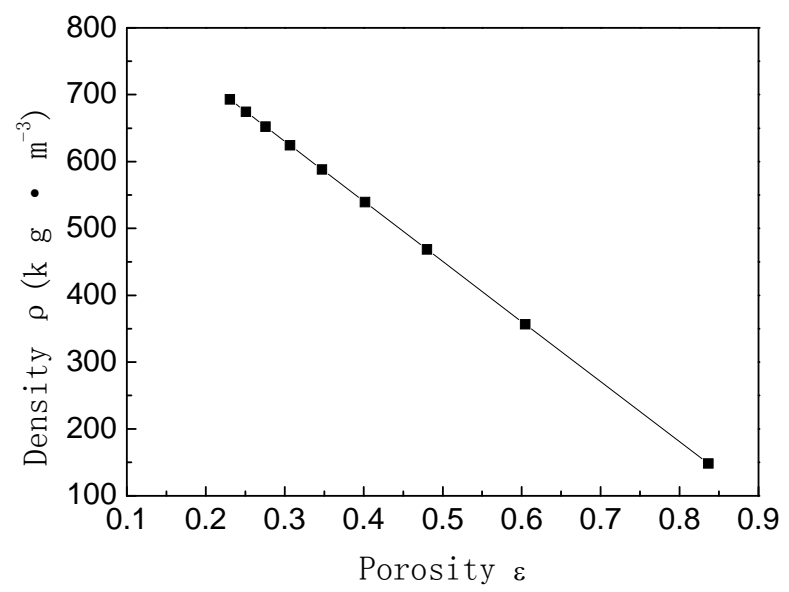

Figure 11. Density varied with the change of porosity.

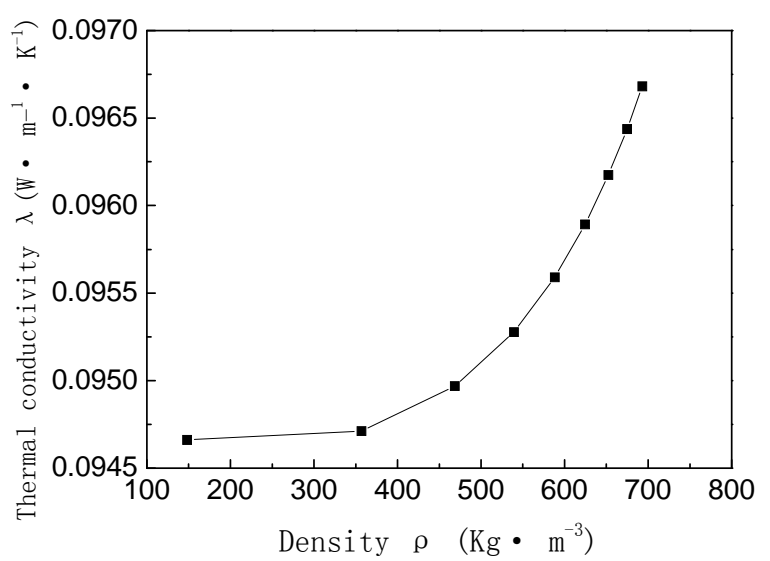

Figure 12. Frost layer thermal conductivity varied with the change of density.

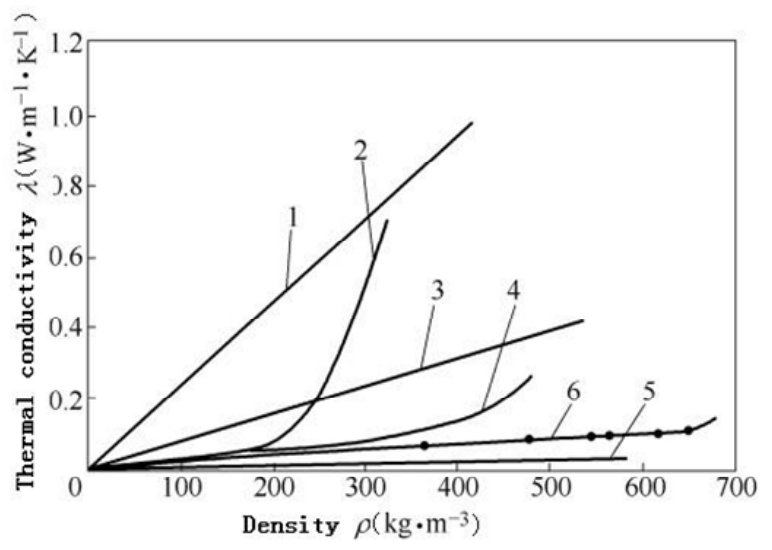

Figure 13. Comparison between the models. 
between each model shown in Figure 13. Therefore, it shows that the thermal conductivity of frost is not only depended on density, but also on the microstructure and frosting environmental conditions. Curve No.1 is obtained from the maximum parallel model and Curve No.5 from the minimum series model. These two curves represent the limits of thermal conductivity of frost, and the curve of thermal conductivity of actual frost should lie between these two curves. Curve No.2 is the random mixture model, which applies to the mixture of air and ice mixed at certain proportion. But it has big deviation from practical case due to the soaring of thermal conductivity under small porosity. The cubic lattice model Curve No.3 consists of open cubic lattice structure, with ice depositing on the edge of the lattice, where the moist air exists between lattices. It can be seen from the Figure 13 that the thermal conductivity of this model is going up rapidly in the low density, not in accordance with the real situation. Yonko-Sepsy model Curve No.4 considers frost as a mixture of ice and air. The calculation result is more practical, but this model is only valid to a certain range of porosity [19]. Curve No.6 is obtained from the model in the present paper. The modeling result lies in between the minimum series model and Yonko-Sepsy model. The model can be applied to the density of frost greater than $500 \mathrm{~kg} \cdot \mathrm{m}^{-3}$, that is, the frost turning into the scale of full growth and not restricted by the porosity. Therefore, the thermal conductivity calculated by the above fractal model coincides with the real situation better than other models, and the thermal conductivity of frost has a wider applicable scale.

\section{Conclusions}

The fractal dimension of pore area distribution and fractal porosity of frost profile were determined based on the observed structure of frost growth. Then, the heat conduction model in frost layer is proposed by using fractal method, and the thermal conductivity is also expressed by a function of the fractal dimension of pore area distribution and porosity of frost. The calculated result shows that thermal conductivity of frost obtained by the fractal model agrees well with the testing data of the effective thermal conductivity. In addition, this fractal model of heat conduction is not restricted by the porosity, and will have broader application area compared to other models. It also verifies the feasibility of adopting the fractal dimension of pore area distribution into the model of heat conduction to determine thermal conductivity. The research shows that frost physical properties is of great importance in evaluating the heat transfer performance of air-heating vaporizer under frosting conditions, which will help optimizing the structure of vaporizer and the defrosting time.

\section{Acknowledgements}

This study was supported by the National Natural Science Foundation of China No. 51076061.

\section{REFERENCES}

[1] H. W. Schneider, "Equation of the Growth Rate of Frost Forming on Cooled Surface," International Journal of Heat and Mass Transfer, Vol. 21, No. 8, 1978, pp. 1019-1024. doi:10.1016/0017-9310(78)90098-4

[2] D. L. O 'Neal and D. R. Tree, "Measurement of Frost Growth and Density in a Parallel Plate Geometry," $A S H$ RAE Transactions, Vol. 26, No. 5, 1984, p. 56.

[3] S. M. Sami and T. Duong, "Mass and Heat Transfer during Frost Growth," ASHRAE Transactions, Vol. 95, No. 1, 1989, pp. 158-165.

[4] D. K. Yang and K. S. Lee, "Dimensionless Correlations of Frost Properties on a Cold Plate," International Journal of Refrigeration, Vol. 27, No. 1, 2004, pp. 89-96. doi:10.1016/S0140-7007(03)00118-X

[5] G. Biguria and L. A. Wenzel, "Measurement and Correlation of Water Frost Thermal Conductivity and Density," Industrial and Engineering Chemistry Fundamentals, Vol. 9, No. 1, 1970, pp. 129-138. doi:10.1021/i160033a021

[6] A. Z. ahin, "Effective Thermal Conductivity of Frost During the Crystal Growth Period," International Journal of Heat and Mass Transfer, Vol. 43, No. 4, 2000, pp. 539-553. doi:10.1016/S0017-9310(99)00162-3

[7] C. H. Cheng and C. H. Shiu, "Frost Formation and Frost Crystal Growth on a Cold Plate in Atmospheric Air Flow," International Journal of Heat and Mass Transfer, Vol. 45, No. 21, 2002, pp. 4289-4303.

[8] P. L. T. Brian, R. C. Reid and Y. T. Shah, "Frost Deposition on Cold Surfaces," Industrial and Engineering Chemistry Fundamentals, Vol. 9, No. 3, 1970, pp. 375-380. doi:10.1021/i160035a013

[9] Y. Hayashi, A. Aoki, S. Adachi and K. Hori, "Study of Frost Properties Correlating with Frost Formation Types," Journal of Heat Transfer, Vol. 99, No. 2, 1977, pp. 239-245. doi:10.1115/1.3450675

[10] Y.-X. Tao, R. W. Besant and K. S. Rezkallah, "A Mathematical Model for Predicting the Densification and Growth of Frost on a Flat Plate," International Journal of Heat and Mass Transfer, Vol. 36, No. 2, 1993, pp. 353-363. doi:10.1016/0017-9310(93)80011-I

[11] R. Le gall, J. M. Grillot and C. Jallut, "Modeling of Frost Growth and Densification," International Journal of Heat and Mass Transfer, Vol. 40, No. 13, 1997, pp. 3177-3187. doi:10.1016/S0017-9310(96)00359-6

[12] H. Chen, R. W. Besant and Y. X. Tao, "Frost Characteristics and Heat Transfer on a Flat Plate under Freezer Operating Conditions, Part II: Numerical Modeling and Comparison with Data," ASHRAE Transactions, Vol. 105, No. 1, 1999, pp. 252-259. 
[13] L. Cai, R. H. Wang, P. X. Hou and X. S. Zhang, "Computer Simulation of Frost Growth and Computation of Its Thermal Conductivity," Chinese Journal of Chemical Engineering, Vol. 60, No. 5, 2009, pp. 1111-1115.

[14] Y. L. Hao, I. Jose and X. T. Yong, "Experimental Study of Initial State of Frost Formation on Flat Surface," Journal of Southeast University, Vol. 35, No. 1, 2005, pp. 149-153.

[15] P. X. Hou, L. Cai and W. P. Yu, "Experimental Study and Fractal Analysis of Ice Crystal Structure at Initial Period of Frost Formation," Journal of Applied Sciences, Vol. 25, No. 2, 2007, pp.193-197.

[16] S. P. Chen, S. T. Yao, F. S. Xie, Z. X. Chang and H. Y.
Han, "Frost Model and Numerical Simulation of Air-heating Fin-tube Vaporizer," Cryogenics \& Superconductivity, Vol. 39, No. 11, 2011, pp. 64-67.

[17] K. Falconer, "Fractal Geometry Mathematical Foundations and Applications," W.Q. Zeng, Transactions, Second edition, Posts \&Telecom Press, Beijing, 2007, pp. 270-275.

[18] B. Na, "Analysis of Frost Formation in an Evaporator," ph.D. Thesis, Pennsylvania State University, 2003.

[19] J. D. Yonko and C. F. Sepsy, "An Investigation of the Thermal Conductivity of Frost while Forming on a Flat Horizontal Plate," ASHRAE Transactions, Vol. 73, No. 2, 1967, pp. 1.1-1.11. 